\title{
Ferromagnetism in multi-band Hubbard models: From weak to strong Coulomb repulsion
}

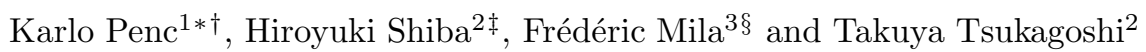 \\ ${ }^{1}$ Max-Planck-Institut für Physik komplexer Systeme, Bayreuther Str. 40, D-01187 Dresden, Germany \\ ${ }^{2}$ Department of Physics, Tokyo Institute of Technology, Oh-Okayama, Tokyo 152, Japan \\ ${ }^{3}$ Laboratoire de Physique Quantique, Univerisité Paul Sabatier, 31062 Toulouse, France
}

(August 8, 2018)

\begin{abstract}
We propose a new mechanism which can lead to ferromagnetism in Hubbard models containing triangles with different on-site energies. It is based on an effective Hamiltonian that we derive in the strong coupling limit. Considering a one-dimensional realization of the model, we show that in the quarter-filled, insulating case the ground-state is actually ferromagnetic in a very large parameter range going from Tasaki's flat-band limit to the strong coupling limit of the effective Hamiltonian. This result has been obtained using a variety of analytical and numerical techniques. Finally, the same results are shown to apply away from quarter-filling, in the metallic case.
\end{abstract}

\section{INTRODUCTION}

Notwithstanding its long history the study of itinerant ferromagnetism is known as one of the most difficult theoretical problems. At the early stage the problem of ferromagnetism was studied within the mean field approximation to the Coulomb interaction. However, in 1960's Kanamori, [1] Gutzwiller [2] and Hubbard [3] independently studied the effect of electron correlations on metallic ferromagnetism, which is left out in the mean field approximation. According to them correlation effects are so important that itinerant ferromagnetism is possible only in restricted situations: a large density of states at the Fermi energy is not sufficient to realize ferromagnetism. Nagaoka [4] and Thouless [5] pointed out a different aspect of ferromagnetism. Studying the motion of a hole (and a few holes) in strongly correlated electrons, Nagaoka and other researchers noticed that the possibility of ferromagnetism is a delicate issue depending on the lattice structure and the density of electrons. [6.7]

Quite recently several exact results have been found for ferromagnetism in Hubbard and related models; [8, 9] However those results are restricted to a special density of electrons. In this connection we note in particular that Tasaki has taken up the problem of ferromagnetism in a series of interesting papers 10 13, and has studied several new models, in which the relevant band is flat or almost flat. For such models he succeeded in proving rigorously that the ground state is ferromagnetic when the electron density takes a particular value, namely $1 / 2$ (quarter-filling), in which case the system is insulating.

The purpose of the present work is to reexamine the same problem from a slightly different viewpoint. Our approach can provide a new way to look at the problem of ferromagnetism and can thereby examine the stability of ferromagnetism, at least in some cases, without any restriction to the electron density.

Restricting ourselves for a while to one-dimensional systems, we will consider a model which interpolates between systems with large density of states and Nagaokalike ferromagnetism, and we focus our attention on the following Hamiltonian:

$$
\begin{aligned}
\mathcal{H}= & s \sum_{j, \sigma}\left(c_{j \sigma}^{\dagger} c_{j+1 \sigma}+\text { H.c. }\right)-t \sum_{j, \sigma}\left(d_{j-\frac{1}{2} \sigma}^{\dagger} d_{j+\frac{1}{2} \sigma}+\text { H.c. }\right) \\
& +t^{\prime} \sum_{j}\left[\left(d_{j-\frac{1}{2} \sigma}^{\dagger}+d_{j+\frac{1}{2} \sigma}^{\dagger}\right) c_{j \sigma}+\text { H.c. }\right]+\varepsilon_{0} \sum_{j} n_{j+\frac{1}{2} \sigma} \\
& +U \sum_{j} n_{j \uparrow} n_{j \downarrow}+U^{\prime} \sum_{j} n_{j+\frac{1}{2} \uparrow} n_{j+\frac{1}{2} \downarrow},
\end{aligned}
$$

where $s$ is assumed to be positive, while $\varepsilon_{0}$ and $t$ are more or less arbitrary and restrictions will be made when needed. Index $j$ takes integer values, and sites with integer index we call ' $c$ ' sites, while those with half-odd integer index 'd' sites. We consider cases where the number of electrons $N_{e}$ is equal or less than the number of 'c' sites $\left(n \leq \frac{1}{2}\right.$, where $n=N_{e} / L$ and $L$ is the total number of sites). The ferromagnetic state has total spin $S=S_{\max }=\frac{1}{2} N_{e}$.

This two band model, which is depicted in Fig. 1, has more degrees of freedom than the regular Hubbard model and it should be able to exhibit more phases. Some special cases has already been studied in literature. For $t=0$ and $\varepsilon_{0}=\varepsilon_{\mathrm{FB}}=t^{\prime 2} / s-2 s$ the dispersion of one of the bands becomes flat, and the ferromagnetism in the so called flat band models has been discussed in the works of Mielke [14] and Tasaki. [10] Moreover, for $\varepsilon_{0}=0$, $U=U^{\prime}$ and $t=-s$ the model is equivalent to what Müller-Hartmann studied in the low density limit. 15. Finally, for $\varepsilon_{0}=0, U=U^{\prime}=+\infty, t=-s$ and $t^{\prime} \ll t$ it was discussed by Long and coworkers. [16]

Let us also point out that by applying a particlehole transformation to this model we get a Hamiltonian where the hopping amplitudes change sign, $\varepsilon_{0}$ becomes $-\varepsilon_{0}+U-U^{\prime}$, the Coulomb interactions are unchanged, and the density transforms as $n \rightarrow 2-n$.

There are several reasons to believe that the ferromagnetic ground state should be stable in Eq. (11) for a rela- 
tively wide range of parameters.

First, the basic building block is a triangle with two ' $c$ ' sites and one 'd' sites, and the triangles are connected to form a chain (one dimensional model), or to form some kind of two (see Fig. 2) or three dimensional lattice. Taking this triangle and putting two electrons on it, the triplet ground state is favored over the singlet for a relatively large region of the hopping $t^{\prime}, \varepsilon_{0}$ and interaction $U$. Especially, if $\varepsilon_{0}=t^{2} / s-s$, then the high spin state is realized for any finite value of $U$.

Second, as we already mentioned, for $\varepsilon_{0}=\varepsilon_{\mathrm{FB}}$ the lower band is flat, and it has been proved that the model is ferromagnetic for any $U>0$ at quarter filling $(n=1 / 2)$. A nearly flat band also means large density of states near the Fermi level.

Third, anticipating the results of Sec. III, we mention that for $U=+\infty$ and large $\varepsilon_{0}$ the usual second order process giving rise to effective antiferromagnetic coupling between spins on neighboring sites in the case of the regular Hubbard model is suppressed. Instead we have a third order process with an effective exchange $\propto-s t^{\prime 2} / \varepsilon_{0}^{2}$, where the sign can be adjusted by the hopping $s$ and it is ferromagnetic for $s>0$. This is also valid away from quarter filling. The mechanism described is clearly of kinetic origin and resembles Nagaoka-effect.

These arguments raise the following questions on the model in Eq. (11):

1) Does the high spin ground state survives if we put many triangles together? In other words, how robust is the Tasaki ferromagnetism against a perturbation which destroys the flatness of the band? And to what extent is the strong coupling perturbational argument valid?

2) Are there any cases in Eq. (1), where metallic ferromagnetism is realized?

\section{SUMMARY OF THE RESULTS}

To motivate the reader's curiosity, let us start by showing in Fig. 3 the phase diagram we got in the insulating, quarter-filled case. We see that ferromagnetism with a correct excitation spectrum turns out to be a robust feature of this model and is realized in a fairly large parameter region. It is not surprising if we recall that in some loose sense our model interpolates between the Nagaoka like ferromagnetism of kinetic origin in the large- $U$ limit and flat band ferromagnetism with large density of states at the Fermi level, and that they both favor the fully polarized, ferromagnetic ground state. This is very encouraging since it shows that ferromagnetism can show up for cases that are more realistic than the flat band model or Nagaoka effect. We emphasize that near flat band models are multi-band systems, with the important consequence that the interaction becomes momentum dependent ( and is not constant as in the one-band
Hubbard models) and this effective long range interaction is essential to stabilize the ferromagnetic state for small values of $U$. Furthermore the role of the triangles is not restricted to generate effective ferromagnetic exchange, but also prevents ferrimagnetism 17 (not being a bipartite lattice) and its importance has been already recognized by Takahashi 18] and Shastry et al. [19]. Unfortunately, for the itinerant case the situation is not so clear. Although the phase diagram is similar to that of the insulating case, the boundary effects due to low dimensionality of the model are enhanced and make the analysis difficult.

Finally, let us remark that although we studied the one-dimensional model in details, we believe that similar conclusions would hold for higher dimensional models as well.

To reach the above conclusions we analyzed the model in Eq. (11) with different techniques in the appropriate limits:

a. Limit of $U=+\infty$ and $\varepsilon_{0} \rightarrow \infty$, any density. In this limit we use a canonical transformation to derive a low energy effective Hamiltonian and we show that ferromagnetic exchange is generated in the third order.

b. Large $U$ and $\varepsilon_{0}$, quarter filling $(n=1 / 2)$ : Rayleigh-Schrödinger perturbation theory allows us to derive a series expansion for the dispersion of the spin flipped state up to 7 th order. The series is then analyzed with the help of Padé approximants and we determined the boundary of the local stability within the radius of convergence. In the lowest order the model is ferromagnetic for $U>U_{c} \approx s \varepsilon_{0}^{2} / t^{\prime 2}$.

c. Small $U$ and nearly flat band, quarter filling. For the cases when both $U$ and the width of the lower band is small comparable to the band gap, we concentrate on the Hamiltonian restricted to states in the lower band. We find that there is a very nice Goldstone mode (magnon) with dispersion $\omega(q)=D q^{2}+\mathcal{O}\left(q^{4}\right)$ for $q \rightarrow 0$, where the spin stiffness $D$ can be calculated analytically. Furthermore, the ferromagnetic state is stable for $U>U_{c} \propto|\rho|$, where $\rho$ measures the flatness of the band and is defined as $\rho=\varepsilon_{0}-\varepsilon_{\mathrm{FB}}$.

d. Quarter filling, arbitrary $U$ and $\varepsilon_{0}$, small size systems. We diagonalize small clusters in the $S^{z}=S_{\max }-1$ (spin flipped) subspace up to 20 sites and in the $S^{z}=0$ subspace up to 16 sites. The data are consistent with the results obtained in the aforementioned limits, and we fill the phase diagram for any $\varepsilon_{0}$. The global stability seems to indicate that the transition is from ferromagnetic to singlet state, except near the flat band where we do not have reliable data due to convergence problems. We find also that near $\varepsilon_{0}=-2 s$ the system is very sensitive to boundary conditions due to orbital degeneracy of the ferromagnetic state.

e. $U=+\infty$ and large $\varepsilon_{0}$, less than quarter filling $(n<1 / 2) \quad$ Using the factorized wave function, we determine the sign of the effective spin-spin interaction for 
different values of $s, t^{\prime}$ and $t$. We discuss the excitation spectrum of the spin flipped state for $J_{\text {eff }} \ll s, t^{\prime}, t$, and show that in case of orbital degeneracy of the ferromagnetic state we need a finite value of $J_{\text {eff }}$ to make the high spin state the lowest in energy.

f. Arbitrary $U$ and $\varepsilon_{0}$, eighth filling $(n=1 / 4)$, small size systems. From exact diagonalization of $L=$ $12,16,20,24$ site systems in the $S^{z}=S_{\max }-1$ subspace with $N_{e}=L / 4$ electrons we determine the boundary of the local stability. Due to large finite size effect the results are not always conclusive. The effect of orbital degeneracy is enhanced compared to the insulating quarter filled system.

\section{LOW-ENERGY EFFECTIVE HAMILTONIAN IN THE STRONG COUPLING LIMIT}

In this section, we will derive an effective Hamiltonian when $t, t^{\prime}, s \ll \varepsilon_{0}, U, U^{\prime}$. In this limit, we have to distinguish between two cases depending on the relative magnitude of $\varepsilon_{0}$ to $U$ and $U^{\prime}: \varepsilon_{0}<U, U^{\prime}$ and $U, U^{\prime}<\varepsilon_{0}$. First we study the former case and we take the large$U$ limit, i.e. $U, U^{\prime} \rightarrow+\infty$. We mainly discuss the case where the electron density satisfies $n \leq \frac{1}{2}$. Since $\varepsilon_{0}$ is positive, only the lower band consisting mostly of the ' $\mathrm{c}$ ' component is filled.
For $U, U^{\prime} \rightarrow \infty$ the Hamiltonian is reduced to

$$
\begin{aligned}
\mathcal{H}= & s \sum_{j, \sigma}\left(\tilde{c}_{j \sigma}^{\dagger} \tilde{c}_{j+1 \sigma}+\text { H.c. }\right)-t \sum_{j, \sigma}\left(\tilde{d}_{j-\frac{1}{2} \sigma}^{\dagger} \tilde{d}_{j+\frac{1}{2} \sigma}+\text { H.c. }\right) \\
& +t^{\prime} \sum_{j, \sigma}\left[\left(\tilde{d}_{j-\frac{1}{2} \sigma}^{\dagger}+\tilde{d}_{j+\frac{1}{2} \sigma}^{\dagger}\right) \tilde{c}_{j \sigma}+\text { H.c. }\right]+\varepsilon_{0} \sum_{j, \sigma} n_{j+\frac{1}{2} \sigma},
\end{aligned}
$$

where $\tilde{c}_{j \sigma}$ and $\tilde{d}_{j-\frac{1}{2} \sigma}$ are annihilation operators under the constraint of no double occupancy: $\tilde{c}_{j \sigma}=c_{j \sigma}\left(1-n_{j \bar{\sigma}}\right)$, $\tilde{d}_{j-\frac{1}{2} \sigma}=d_{j-\frac{1}{2} \sigma}\left(1-n_{j-\frac{1}{2} \bar{\sigma}}\right)$ and $\bar{\sigma}=-\sigma$. The creation operators are defined similarly.

To derive an effective Hamiltonian to describe lowenergy physics, we can treat the $t^{\prime} \ll \varepsilon_{0}$ term as a perturbation inducing transitions between the lower and upper bands. We use the canonical transformation to eliminate the off-diagonal processes due to

$$
\mathcal{H}^{\prime}=t^{\prime} \sum_{j \sigma}\left[\left(\tilde{d}_{j-\frac{1}{2} \sigma}^{\dagger}+\tilde{d}_{j+\frac{1}{2} \sigma}^{\dagger}\right) \tilde{c}_{j \sigma}+\text { H.c. }\right] \text {. }
$$

The other terms in Eq. (2) are denoted as $\mathcal{H}_{0}$. Since the canonical transformation is standard, we skip the details. Eliminating the first-order effect of $t^{\prime}$, an effective Hamiltonian up to the second order in $t^{\prime}$ is obtained as follows:

$$
\left\langle m^{\prime}\left|\mathcal{H}_{\mathrm{eff}}\right| m\right\rangle=\left\langle m\left|\mathcal{H}_{0}\right| m\right\rangle \delta_{m m^{\prime}}+\frac{1}{2} \sum_{n}\left(\frac{\left\langle m^{\prime}\left|\mathcal{H}^{\prime}\right| n\right\rangle\left\langle n\left|\mathcal{H}^{\prime}\right| m\right\rangle}{E_{m}-E_{n}}+\frac{\left\langle m^{\prime}\left|\mathcal{H}^{\prime}\right| n\right\rangle\left\langle n\left|\mathcal{H}^{\prime}\right| m\right\rangle}{E_{m^{\prime}}-E_{n}}\right),
$$

where $m, m^{\prime}, n$ are eigenstates of $\mathcal{H}_{0}$. The energy denominator $E_{m}-E_{n}$ can be written as $E_{m}-E_{n}=-\varepsilon_{0}+E_{m}^{\prime}-E_{n}^{\prime}$. Here $E_{m}^{\prime}$ represents the part due to $t$ and $s$ in the eigenvalue $E_{m}$, i.e. $\mathcal{H}_{0}^{\prime}|m\rangle=E_{m}^{\prime}|m\rangle$ where $\mathcal{H}_{0}^{\prime}$ denotes $\mathcal{H}_{0}$ except for the $\varepsilon_{0}$ term. Since $\varepsilon_{0}$ is assumed to be much larger than $t$ and $s$, we can expand the energy denominators in the second term of Eq. (4) in terms of $\mathcal{H}_{0}^{\prime}$ as

$$
\begin{aligned}
\left\langle m^{\prime}\left|\mathcal{H}_{\mathrm{eff}}\right| m\right\rangle= & \left\langle m\left|\mathcal{H}_{0}\right| m\right\rangle \delta_{m m^{\prime}}-\frac{1}{\varepsilon_{0}}\left\langle m^{\prime}\left|\mathcal{H}^{\prime} \mathcal{H}^{\prime}\right| m\right\rangle+\frac{1}{2 \varepsilon_{0}^{2}}\left(\left\langle m^{\prime}\left|\mathcal{H}^{\prime}\left[\mathcal{H}_{0}^{\prime}, \mathcal{H}^{\prime}\right]\right| m\right\rangle+\left\langle m^{\prime}\left|\left[\mathcal{H}^{\prime}, \mathcal{H}_{0}^{\prime}\right] \mathcal{H}^{\prime}\right| m\right\rangle\right) \\
& -\frac{1}{2 \varepsilon_{0}^{3}}\left(\left\langle m^{\prime}\left|\mathcal{H}^{\prime}\left[\mathcal{H}_{0},\left[\mathcal{H}_{0}^{\prime}, \mathcal{H}^{\prime}\right]\right]\right| m\right\rangle+\left\langle m^{\prime}\left|\left[\left[\mathcal{H}^{\prime}, \mathcal{H}_{0}^{\prime}\right], \mathcal{H}_{0}^{\prime}\right] \mathcal{H}^{\prime}\right| m\right\rangle\right)+\cdots
\end{aligned}
$$

As mentioned before, we assume $n \leq \frac{1}{2}$. Then only the lower band is filled. Having such states in mind, we evaluate each term in Eq. (5). The result is as follows:

$$
\begin{aligned}
& \mathcal{H}_{\mathrm{eff}}^{(1)}=s \sum_{j \sigma}\left(\tilde{c}_{j \sigma}^{\dagger} \tilde{c}_{j+1 \sigma}+\text { H.c. }\right), \\
& \mathcal{H}_{\mathrm{eff}}^{(2)}=-\frac{t^{\prime 2}}{\varepsilon_{0}} \sum_{j \sigma}\left(2 n_{j \sigma}^{\dagger}+\tilde{c}_{j-1 \sigma}^{\dagger} \tilde{c}_{j \sigma}+\tilde{c}_{j+1 \sigma}^{\dagger} \tilde{c}_{j \sigma}\right),
\end{aligned}
$$

and $\mathcal{H}_{\text {eff }}^{(3)}=\mathcal{H}_{\text {eff }}^{(3 a)}+\mathcal{H}_{\text {eff }}^{(3 b)}$ with

$$
\mathcal{H}_{\mathrm{eff}}^{(3 a)}=-\frac{t t^{\prime 2}}{\varepsilon_{0}^{2}} \sum_{j \sigma}\left[2 n_{j \sigma}+2\left(\tilde{c}_{j-1 \sigma}^{\dagger}+\tilde{c}_{j+1 \sigma}^{\dagger}\right) \tilde{c}_{j \sigma}\right.
$$

$$
\begin{aligned}
& \left.+\left(\tilde{c}_{j-2 \sigma}^{\dagger}+\tilde{c}_{j+2 \sigma}^{\dagger}\right) \tilde{c}_{j \sigma}\right] \\
\mathcal{H}_{\mathrm{eff}}^{(3 b)}= & -\frac{s t^{\prime 2}}{\varepsilon_{0}^{2}} \sum_{j \sigma}\left[2 \tilde{c}_{j \sigma}^{\dagger}\left(\tilde{c}_{j-1 \sigma}+\tilde{c}_{j+1 \sigma}\right)\right. \\
& +\left(n_{j-1 \sigma}+n_{j+1 \sigma}\right)\left(1-n_{j \bar{\sigma}}\right) \\
& +\left(\tilde{c}_{j-1 \sigma}^{\dagger} \tilde{c}_{j-1 \bar{\sigma}}+\tilde{c}_{j+1 \sigma}^{\dagger} \tilde{c}_{j+1 \bar{\sigma}}\right) \tilde{c}_{j \bar{\sigma}}^{\dagger} \tilde{c}_{j \sigma} \\
& +\tilde{c}_{j-1 \sigma}^{\dagger}\left(1-n_{j \bar{\sigma}}\right) \tilde{c}_{j+1 \sigma}+\tilde{c}_{j+1 \sigma}^{\dagger}\left(1-n_{j \bar{\sigma}}\right) \tilde{c}_{j-1 \sigma} \\
& \left.+\left(\tilde{c}_{j-1 \sigma}^{\dagger} \tilde{c}_{j+1 \bar{\sigma}}+\tilde{c}_{j+1 \sigma}^{\dagger} \tilde{c}_{j-1 \bar{\sigma}}\right) \tilde{c}_{j \bar{\sigma}}^{\dagger} \tilde{c}_{j \sigma}\right]
\end{aligned}
$$

Although it looks complicated, Eqs. (6)-(9) become simple for $n=\frac{1}{2}$, in which the states $|m\rangle$ are written as 


$$
|m\rangle=\prod_{j} \tilde{c}_{j \sigma(j)}^{\dagger}|\operatorname{vacuum}\rangle
$$

where the product is taken over all integer sites and where the quantum numbers $m$ just correspond to the set of spin indices $\sigma(j)$. In fact it is easy to see that except for constant terms $\mathcal{H}_{\text {eff }}$ is reduced to

$$
\mathcal{H}_{\mathrm{eff}}=-4 s\left(\frac{t^{\prime}}{\varepsilon_{0}}\right)^{2} \sum_{j}\left(\mathbf{S}_{j} \cdot \mathbf{S}_{j+1}-\frac{1}{4}\right),
$$

where $\mathbf{S}_{j}$ means a spin $\frac{1}{2}$ at site $j$. Eq. (11) shows that the effective interaction is third order in the hopping; it is essentially a ferromagnetic Heisenberg model for positive $s$. Therefore the ground state is ferromagnetic with the maximum total spin. The reason for this result is simple. The contribution in Eq. (11) comes from the ring exchange among 3 sites (a triangle formed with $j, j+1, j+\frac{1}{2}$ sites, see Fig. (1) with the hopping having the same sign, which is known to lead to a ferromagnetic exchange. Needless to say the system is insulating with a charge gap of $\sim \varepsilon_{0}$. Here we note that although Eq. (11) has been derived for the one-dimensional case, it is applicable to more general cases like the ones shown in Fig. 2 . In that case the summation over $j$ should be interpreted as the one for all the nearest neighbor 'c' sites.

So far we have assumed $U, U^{\prime} \rightarrow \infty$. Relaxing this condition, let us take into account the lowest term in $1 / U$. This is essentially the same as the one-band Hubbard model in the large- $U$ limit. [20] The effective Hamiltonian which should be added to Eqs. (6-9) is

$$
\begin{aligned}
\mathcal{H}_{\mathrm{eff}}^{\prime}= & \frac{4 s^{2}}{U} \sum_{i}\left(\mathbf{S}_{i} \cdot \mathbf{S}_{i+1}-\frac{1}{4} n_{i} n_{i+1}\right) \\
& +\frac{s^{2}}{U} \sum_{i, \sigma}\left(\tilde{c}_{i, \sigma}^{\dagger} \tilde{c}_{i+1, \bar{\sigma}}^{\dagger} \tilde{c}_{i+1, \sigma} \tilde{c}_{i+2, \bar{\sigma}}\right. \\
& \left.-\tilde{c}_{i, \sigma}^{\dagger} n_{i+1, \bar{\sigma}} \tilde{c}_{i+2, \sigma}+\text { H.c. }\right),
\end{aligned}
$$

where $n_{j}=\sum_{\sigma} n_{j \sigma}$ is the density of electrons at site $j$, and for quarter filling it simplifies to

$$
\mathcal{H}_{\mathrm{eff}}^{\prime}=\frac{4 s^{2}}{U} \sum_{i}\left(\mathbf{S}_{i} \cdot \mathbf{S}_{i+1}-\frac{1}{4}\right) .
$$

This Hamiltonian favors antiferromagnetic fluctuations and works against ferromagnetism, so the final state will be decided by the competition between this Hamiltanion and that of Eq. (9).

\section{STABILITY OF FERROMAGNETISM AT QUARTER-FILLING $(n=1 / 2)$}

We now examine how robust ferromagnetism is at quarter-filling. We will look mainly at the stability against one spin flip; this is the local stability condition. At some points we will mention the global stability as well. Henceforth we assume, for simplicity, $U^{\prime}=U$ and $t=0$ in Eq. (1), in which case the topology of the model is the $\Delta$ chain.

\section{A. Large $U$ and $\varepsilon_{0}$}

As we noted in the previous section, for $\varepsilon_{0}$ positive, and for $U$ and $\varepsilon_{0}$ much larger than the hopping amplitudes, the quarter filled system exhibits a charge gap 21] $\Delta_{c} \approx \min \left(U, \varepsilon_{0}\right)$. Every 'c' site is occupied by one electron, the excitations in the charge sector are pushed up to high energies, and the only low lying excitations are coming from the spin degrees of freedom, which can be described in lowest order by the competition of Hamiltonians (11) and (13).

To get reliable results in this limit, it is desirable to extend the calculation of the effective spin Hamiltonian to higher order corrections. Although in principle it can be done, see for example in Ref. [22], it is a very difficult task, especially for a relatively complicated model as in Eq. (11). Therefore here we follow a slightly different approach, and we compute the dispersion relation of the spin flipped state given by

$$
\varepsilon(k)=-K_{1}[1-\cos (k)]-K_{2}[1-\cos (2 k)]-\ldots,
$$

where the coefficients are related to the exchange integrals in the spin Hamiltonians. Were the spin Hamiltonian of the form $J_{1} \sum_{i} \mathbf{S}_{i} \cdot \mathbf{S}_{i+1}+J_{2} \sum_{i} \mathbf{S}_{i} \cdot \mathbf{S}_{i+2}+\ldots$ then $K_{1}=J_{1}, K_{2}=J_{2}$ would hold. However, the canonical transformation generates terms 22 like $\left(\mathbf{S}_{i} \mathbf{S}_{i^{\prime}}\right)\left(\mathbf{S}_{j} \mathbf{S}_{j^{\prime}}\right)$ etc., and this makes the correspondence difficult.

The series expansion for the coefficients in Eq. (14) can be obtained using the standard Rayleigh-Schrödinger perturbation theory. As an initial state one picks up a state where all the 'c' sites are occupied with spin up electrons except for one site, where the spin points downwards, furthermore all the 'd' sites are empty. Since the system is translationally invariant, momentum is a good quantum number, and the problem is simplified as the Hilbert space is spanned by states with given momentum $k$. In this way we avoid the $L / 2$ fold degeneracy of the ground state, as in every $k$ subspace the lowest energy state is unique. Now, through a number of virtual processes (hopping to 'd' sites, or hopping to already occupied sites), the spin down electron will finally return to a 'c' site. The distance $j$ which the down spin electron has traveled will pick up a phase factor $e^{i k j}$ and will contribute to $K_{j}$. Although it does not look difficult, the technical realization of this calculation for higher orders is not straightforward. We used a variation of the method given by Barber and Duxbury, [23] which is relatively simple. Recently, a more efficient method based on 
linked cluster expansion become available due to Gelfand. 24

We have calculated the coefficients $K_{1}, K_{2}, \ldots$ up to 7th order, and they are given in the Appendix. The lowest order contributions are

$$
\begin{aligned}
& K_{1}^{(2)}=\frac{4 s^{2}}{U}, \\
& K_{1}^{(3)}=-\frac{4 s t^{\prime 2}}{\varepsilon_{0}^{2}}-\frac{8 s t^{\prime 2}}{U \varepsilon_{0}},
\end{aligned}
$$

in agreement with Eqs. (11) and (13).

The first contribution to $K_{2}$ comes in 4 th order,

$$
\begin{aligned}
K_{2}^{(4)} & =\frac{4 s^{4}}{U^{3}}, \\
K_{2}^{(5)} & =-\frac{16 s^{3} t^{\prime 2}}{\varepsilon_{0} U^{3}}-\frac{8 s^{3} t^{\prime 2}}{\varepsilon_{0}^{2} U^{2}},
\end{aligned}
$$

and $K_{3}$ appears first in 6th order (see Appendix for details).

To be more specific, let us now turn to the special case of $t^{\prime}=s$. The series can be analyzed with Padé approximants. A typical plot of $K_{1}$ as a function of $s / \varepsilon_{0}$ is given in Fig. 5. Fixing the ratio $U / \varepsilon_{0}$, we look for which value of $\varepsilon_{0}$ the coefficient $K_{1}$ changes sign. It is enough to consider $K_{1}$ since $\left|K_{2}\right| \ll\left|K_{1}\right|$ holds near the transition. Changing the ratio of $U / \varepsilon_{0}$, we are able to determine the region where ferromagnetism is locally stable. The result is shown in Fig. 6. For $s \neq t^{\prime}$ there are some quantitative changes in the location of the boundary, which can be already recognized from $K_{1}^{(2)}$ and $K_{1}^{(3)}$ yielding $U_{c} \approx s \varepsilon_{0}^{2} / t^{\prime 2}$.

A similar calculation can be carried over for $U,-\varepsilon_{0} \gg$ $s, t$ '. Then the electrons sit on 'd' sites and for $t=0$ the first contributions to $\bar{K}_{1}$ come in fourth order:

$$
\begin{aligned}
& \bar{K}_{1}^{(4)}=\frac{4 \tilde{t}^{4}}{\bar{\varepsilon}_{0}^{2} U}+\frac{8 \tilde{t}^{4}}{\bar{\varepsilon}_{0}^{2}\left(2 \bar{\varepsilon}_{0}+U\right)}, \\
& \bar{K}_{1}^{(5)}=-\frac{8 \tilde{t}^{5}}{\bar{\varepsilon}_{0}^{4}}-\frac{16 \tilde{t}^{5}}{\bar{\varepsilon}_{0}^{3} U}-\frac{32 \tilde{t}^{5}}{\bar{\varepsilon}_{0}^{3}\left(2 \bar{\varepsilon}_{0}+U\right)} .
\end{aligned}
$$

where we concentrated on $s=t^{\prime}=\tilde{t}$ case, $\bar{\varepsilon}_{0}=-\varepsilon_{0}$ and the coefficients are denoted $\bar{K}_{j}$. For higher order correction up to 7 th order we refer the reader to the Appendix. The series is convergent for $\left|\varepsilon_{0}\right| / \tilde{t} \gtrsim 15$.

\section{B. In the vicinity of the flat band}

Our model is equivalent to Tasaki's flat band model [10] for $\varepsilon_{0}=\varepsilon_{\mathrm{FB}}=t^{\prime 2} / s-2 s$, where he has rigorously proven that the model is ferromagnetic for arbitrarily small value of interaction. In this section we restrict $\varepsilon_{0}$ to be close to $\varepsilon_{\mathrm{FB}}$, and we introduce a quantity $\rho$ which measures the perturbation from the flat band as
$\rho=\varepsilon_{0}-\varepsilon_{\mathrm{FB}}$. In a series of recent papers [11] Tasaki extended the existence of ferromagnetism for such perturbed models, reaching the result that ferromagnetism is stable for the nearly flat bands as well for $U>U_{c}$, where $U_{c}$ is roughly proportional to the perturbation strength, in our case $\rho$. His treatment is rather involved and we are looking for a simpler description.

Let us consider the model Hamiltonian in $k$ space:

$$
\begin{aligned}
\mathcal{H}= & 2 s \sum_{k, \sigma} \cos k c_{k \sigma}^{\dagger} c_{k \sigma}+\varepsilon_{0} \sum_{k, \sigma} d_{k \sigma}^{\dagger} d_{k \sigma} \\
& +2 t^{\prime} \sum_{k, \sigma} \cos \frac{k}{2}\left(c_{k \sigma}^{\dagger} d_{k \sigma}+d_{k \sigma}^{\dagger} c_{k \sigma}\right) \\
& +\frac{2 U}{L} \sum_{k_{1}, k_{2}, k_{3}, k_{4}}\left(c_{k_{1} \uparrow}^{\dagger} c_{k_{2} \downarrow}^{\dagger} c_{k_{3} \downarrow} c_{k_{4} \uparrow}+d_{k_{1} \uparrow}^{\dagger} d_{k_{2} \downarrow}^{\dagger} d_{k_{3} \downarrow} d_{k_{4} \uparrow}\right),
\end{aligned}
$$

where in the summation $k_{1}+k_{2}-k_{3}-k_{4}=G$ should hold, $G=0, \pm 2 \pi$ is a reciprocical lattice vector. In the noninteracting case $(U=0)$ it describes two bands where the lowest band is nearly flat (the bandwidth is $\propto \rho$ ), while the upper band is dispersive. The two bands are separated by a band gap $\Delta_{\text {band }}=2 s+\varepsilon_{0}+\mathcal{O}(\rho)$. We expect, as already noted by Kusakabe and Aoki, 13 that for small values of $U$ the essential physics is now going on in the lower band. To construct an effective Hamiltonian, we first diagonalize the hopping part of the Hamiltonian by the following canonical transformation (here we choose $t^{\prime}>0$ for convenience, as the model does not depend on the sign of $t^{\prime}$ ):

$$
\begin{aligned}
& c_{k \sigma}^{\dagger}=\cos \alpha_{k} a_{k \sigma}^{\dagger}+\sin \alpha_{k} b_{k \sigma}^{\dagger}, \\
& d_{k \sigma}^{\dagger}=-\sin \alpha_{k} a_{k \sigma}^{\dagger}+\cos \alpha_{k} b_{k \sigma}^{\dagger},
\end{aligned}
$$

where $a_{k, \sigma}^{\dagger}$ and $b_{k, \sigma}^{\dagger}$ are the creation operators of electrons on the lower and upper band, respectively. $\alpha_{k}$ is determined via

$$
\tan 2 \alpha_{k}=\frac{4 t^{\prime} \cos (k / 2)}{\varepsilon_{0}-2 s \cos (k)},
$$

and is a continuous function of the momentum $k$, so that it is between 0 and $\pi / 2$ for momenta in the Brillouin zone; furthermore $\alpha_{ \pm \pi}=0$ for $\varepsilon_{0}>-2 s$ and $\alpha_{ \pm \pi}=\frac{\pi}{2}$ for $\varepsilon_{0}<-2 s$. It is convenient to extend its definition outside the Brillouin zone so that $\alpha_{2 \pi+k}=-\alpha_{k}$ for any $k$ in case of $\varepsilon_{0}>-2 s$ and $\alpha_{2 \pi+k}=\pi-\alpha_{k}$ for $\varepsilon_{0}<-2 s$ . The band dispersion is then given by

$$
\varepsilon_{a, b}(k)=\frac{\varepsilon_{0}}{2}+s \cos k \mp \sqrt{\left(\frac{\varepsilon_{0}}{2}-s \cos k\right)^{2}+4 t^{\prime 2} \cos ^{2} \frac{k}{2}} ;
$$

the effective Hamiltonian in the lower band reads 


$$
\begin{aligned}
H= & \sum_{k, \sigma} \varepsilon_{a}(k) a_{k \sigma}^{\dagger} a_{k \sigma} \\
& +\sum_{k_{1}, k_{2}, k_{3}, k_{4}} V\left(k_{1}, k_{2}, k_{3}, k_{4}\right) a_{k_{1} \uparrow}^{\dagger} a_{k_{2} \downarrow}^{\dagger} a_{k_{3} \downarrow} a_{k_{4} \uparrow},
\end{aligned}
$$

where the interaction is defined as

$$
\begin{array}{r}
V\left(k_{1}, k_{2}, k_{3}, k_{4}\right)=\frac{2 U}{L}\left(\cos \alpha_{k_{1}} \cos \alpha_{k_{2}} \cos \alpha_{k_{3}} \cos \alpha_{k_{4}}\right. \\
\left.+e^{i G / 2} \sin \alpha_{k_{1}} \sin \alpha_{k_{2}} \sin \alpha_{k_{3}} \sin \alpha_{k_{4}}\right)
\end{array}
$$

and $k_{1}+k_{2}-k_{3}-k_{4}=G$ holds. The factor $e^{i G / 2}$ is 1 for normal scattering $(G=0)$ and -1 for umklapp scattering $(G=2 \pi)$, and it can be conveniently taken care of using the definition of $\alpha_{k}$ for $k$ values outside the Brillouin zone.

Here we neglected terms which are coming from the scattering on the states in the upper band [21] and they are of the order of $U^{2} / \Delta_{\text {band }}$, so that the approximation is valid for $U \ll \Delta_{\text {band }}$.

We now concentrate on the excitation spectrum of the spin flipped states of momentum $\mathrm{q}$ which are defined as

$$
|\chi(q, \nu)\rangle=\sum_{p} f_{p}^{\nu}(q) a_{p+q, \downarrow}^{\dagger} a_{p, \uparrow}|\mathrm{FP}\rangle,
$$

where $|\mathrm{FP}\rangle$ is the fully polarized state with all the spins up (i.e. $S=S_{\max }=L / 2$ ), $q$ is measured from the momentum of $|\mathrm{FP}\rangle$ and $\nu$ is some quantum number. Since the lower band is filled, these states span the whole reduced Hilbert space and no approximation is made in solving the effective Hamiltonian (22).

The dispersion of the spin flipped state $\omega(q)$ is given by the eigenvalue problem 13.

$$
\sum_{p^{\prime}} M_{p, p^{\prime}}(q) f_{p^{\prime}}^{\nu}(q)=\omega_{\nu}(q) f_{p}^{\nu}(q)
$$

where the matrix is given by

$$
\begin{aligned}
M_{p, p^{\prime}}(q)= & {\left[\varepsilon_{a}(p+q)+\Delta(p+q)-\varepsilon_{a}(p)\right] \delta_{p, p^{\prime}} } \\
& -V\left(p, p^{\prime}+q, p+q, p^{\prime}\right) .
\end{aligned}
$$

$\Delta(p)=\sum_{k} V(k, p, p, k)$ is the Stoner gap. The size of the matrix is $(L / 2) \times(L / 2)$ and can be easily diagonalized numerically for large system sizes.

A typical plot of the excitation spectra is given in Figs. 7 and 8. There we can see that a low energy magnon (Goldstone mode) and an optical magnon (with spin oscillation within the cell) emerge from the Stoner continuum. For small values of $q$ the magnon has a dispersion $\omega_{0}(q)=D q^{2}$.

Now, what are the conditions for local stability? First of all, to have a dispersion relation appropriate for ferromagnets, stiffness $D$ should be positive. The interaction strength where $D$ changes sign we call $U_{D}$. However, this is not enough, as it can happen that although $D>0$, the
Stoner continuum pushes down the spin wave mode at $q=\pi$. Therefore the decisive criterion for local stability is that all the excitation energies are positive (i.e. all the energies are above the energy of the fully polarized state), and this will define $U_{c}$.

\section{Calculation of the $U_{D}$}

Clearly, $D$ is given as $\frac{1}{2} \partial^{2} \omega_{0}(q) /\left.\partial q^{2}\right|_{q=0}$ and it can be calculated from Eq. (26) by using Hellman-Feynman rule (it will give $D_{1}$ ) and second order perturbation theory $\left(D_{2}\right)$, so that $D=D_{1}+D_{2}$, where

$$
\begin{aligned}
& D_{1}=\left.\frac{1}{2}\left\langle\chi(0,0)\left|\frac{\partial^{2} M(q)}{\partial q^{2}}\right| \chi(0,0)\right\rangle\right|_{q=0}, \\
& D_{2}=-\left.\sum_{\nu \neq 0} \frac{1}{\omega_{\nu}-\omega_{0}}\left|\left\langle\chi(0, \nu)\left|\frac{\partial M(q)}{\partial q}\right| \chi(0,0)\right\rangle\right|^{2}\right|_{q=0} .
\end{aligned}
$$

Here $|\chi(0,0)\rangle$ is the ground state wave function for momentum $q=0$, which is nothing else but $S^{-}|\mathrm{FP}\rangle$ and is trivially given by $f_{p}^{0}(0)=\sqrt{2 / L}$. The $|\chi(0, \nu)\rangle$ are excited states. Since by definition $D_{2}$ is negative and works against ferromagnetism, sufficiently large $D_{1}$ is needed to compensate $D_{2}$ and to give $D>0$.

Nonzero contribution to $D_{1}$ will come from the offdiagonal matrix elements of $M_{p, p^{\prime}}$ :

$$
D_{1}=-\left.\frac{1}{2} \int_{-\pi}^{\pi} \frac{d p}{2 \pi} \int_{-\pi}^{\pi} \frac{d p^{\prime}}{2 \pi} \frac{\partial^{2}}{\partial q^{2}} V\left(p, p^{\prime}+q, p+q, p^{\prime}\right)\right|_{q=0}
$$

since the diagonal part vanishes being an integral of the derivative of periodic function. Using the special form of interaction like in Eq. (23) and carrying out partial integration, one can show that this quantity is positive, unless $V$ is a constant. In other words the momentum dependence of the interaction is crucial to stabilize the ferromagnetic state.

Let us now turn to $D_{2}$. In our case the nonvanishing matrix elements come from odd-momentum excited states labeled by momentum $\tilde{p}$, where $f_{p}^{\tilde{p}}(0)=$ $\left(\delta_{p, \tilde{p}}-\delta_{p,-\tilde{p}}\right) / \sqrt{2}$ with energies $\omega_{\nu}-\omega_{0}=\Delta(\tilde{p})$. Thus we obtain

$$
\begin{aligned}
D_{2} & =-\frac{1}{4} \frac{2}{L} \sum_{\tilde{p}} \frac{1}{\Delta(\tilde{p})}\left[\left.\sum_{p} \frac{\partial}{\partial q}\left(M_{p, \tilde{p}}-M_{-p, \tilde{p}}\right)\right|_{q=0}\right]^{2} \\
& =-\frac{1}{4} \int_{-\pi}^{\pi} \frac{d \tilde{p}}{2 \pi} \frac{1}{\Delta(\tilde{p})}\left[2 \frac{\partial \varepsilon(\tilde{p})}{\partial \tilde{p}}+\frac{\partial \Delta(\tilde{p})}{\partial \tilde{p}}\right]^{2}
\end{aligned}
$$

The divergence of $D_{2}$ for small values of $U$ is the signature that the dispersion becomes linear in $q$ for $U=0$ and the result is non-perturbative in $U$.

To make the calculation simple we expand everything in powers of the small parameter $\rho$ : 


$$
\begin{aligned}
\cos \alpha_{k} & =\frac{t^{\prime}}{\sqrt{t^{\prime 2}+4 s^{2} \cos ^{2}(k / 2)}}+\mathcal{O}(\rho), \\
\sin \alpha_{k} & =\frac{2 s \cos (k / 2)}{\sqrt{t^{\prime 2}+4 s^{2} \cos ^{2}(k / 2)}}+\mathcal{O}(\rho), \\
\varepsilon(k) & =-2+\left(1-\cos ^{2} \alpha_{k}\right) \rho+\mathcal{O}\left(\rho^{2}\right) .
\end{aligned}
$$

The following integrals are useful for our purpose:

$$
\begin{aligned}
& I_{c}=\int_{-\pi}^{\pi} \frac{d k}{2 \pi} \cos ^{2} \alpha_{k}=\frac{t^{\prime}}{\sqrt{t^{\prime 2}+4 s^{2}}}, \\
& I_{s}=\int_{-\pi}^{\pi} \frac{d k}{2 \pi} \sin ^{2} \alpha_{k}=1-I_{c}, \\
& I_{c}^{\prime}=\int_{-\pi}^{\pi} \frac{d k}{2 \pi}\left(\frac{d \cos \alpha_{k}}{d k}\right)^{2}=\frac{s^{4}}{2 t^{\prime}\left(t^{\prime 2}+4 s^{2}\right)^{3 / 2}}, \\
& I_{s}^{\prime}=\int_{-\pi}^{\pi} \frac{d k}{2 \pi}\left(\frac{d \sin \alpha_{k}}{d k}\right)^{2}=\frac{s^{2}}{2 t^{\prime} \sqrt{t^{\prime 2}+4 s^{2}}}-I_{c}^{\prime} .
\end{aligned}
$$

With the help of these integrals, we can write the Stoner gap as

$$
\Delta(k)=U\left(I_{c} \cos ^{2} \alpha_{k}+I_{s} \sin ^{2} \alpha_{k}\right) .
$$

Finally, stiffness $D$ is the sum of the $D_{1}$ and $D_{2}$ :

$$
\begin{aligned}
& D_{1}=U\left(I_{c} I_{c}^{\prime}+I_{s} I_{s}^{\prime}\right), \\
& D_{2}=-\frac{C}{U}\left[-2 \rho+\left(2 I_{c}-1\right) U\right]^{2},
\end{aligned}
$$

where

$$
C=\int_{-\pi}^{\pi} \frac{d k}{2 \pi} \frac{\cos ^{2} \alpha_{k}}{I_{c} \cos ^{2} \alpha_{k}+I_{s} \sin ^{2} \alpha_{k}}\left(\frac{\partial \cos \alpha_{k}}{\partial k}\right)^{2} .
$$

Although the coefficient $C$ can be given in a closed form, it is rather complicated and we give its value for some selected values of $t^{\prime} / s$ in Table. I, together with the values of $U$ where $D$ changes $\operatorname{sign}\left(U_{D}\right)$. Note that the equation we solve is a quadratic one and it gives two solutions: one is for $\rho>0$ and the other one is for $\rho<0$.

In the limit $t^{\prime} \gg s$ we get

$$
D=\left(U+2 \rho-2 \frac{\rho^{2}}{U}\right)\left(\frac{s}{t^{\prime}}\right)^{4}
$$

for small $U$ and $\rho$, which is in agreement with Tasaki's result. 11]

\section{Calculation of $U_{c}$}

From numerical diagonalization of the matrix $M_{p, p^{\prime}}$ we see that the other local minimum of the acoustic magnon branch is at $q=\pi$. Unfortunately we do not know how to get $U_{c}$, therefore we have to solve the eigenvalue problem of Eq. (25) numerically and check for which values of $U \omega(q=\pi)>0$ holds. From this we obtain $U_{c}$. Again, we have to distinguish between $\rho>0$ and $\rho<0$. The results are presented in Table. If.

We also learn that for small $t^{\prime}$ a very good estimate of $U_{c}$ can be obtained by using only the diagonal elements of matrix $M_{p, p^{\prime}}(\pi)$ applying the Stoner criterion. Namely, the spin down electron occupies states in an effective band $\varepsilon_{\downarrow}(p)=\varepsilon_{a}(p)+\Delta(p)$ due to the background of spin up electrons. This band is higher in energy than the $\varepsilon_{\uparrow}(p)=\varepsilon_{a}(p)$ of the spin up electrons. According to Stoner's theory, when lowering $U$ the Stoner gap decreases, and at some point we start to fill the band of spin down electrons. If we neglect the off diagonal matrix elements describing the interaction between the hole in the spin up band and the spin down electron, this happens exactly when the energies of the two bands start to overlap. For $\rho>0$ the minimum of $\varepsilon_{\downarrow}(p)$ is at $p=\pi$ and the maximum of $\varepsilon_{\uparrow}(p)$ is at $p=0$, so that the criterion is $\varepsilon_{\downarrow}(\pi)=\varepsilon_{\uparrow}(0)$. This will give us $U_{c}^{\text {app }}=4 \rho /\left(t^{\prime} \sqrt{t^{\prime 2}+4 s^{2}}\right)$; for $\rho<0$ the corresponding equation is $\varepsilon_{\downarrow}(0)=\varepsilon_{\uparrow}(\pi)$.

For $t^{\prime} \gg s$, on the other hand, $U_{D}$ and $U_{c}$ are very close to each other. This is due to the fact that the excited states in this case are far from the acoustic magnon branch and their interference is small for values of $U$ close to $U_{c}$. This was also predicted by Tasaki [1] in the sense that lower and upper bound on the dispersion relation are very close to each other in this case.

We have compared these results with the exact diagonalization results for the stability of the spin flipped state in Fig. 9 for $t^{\prime}=s$ and we found that $U_{c}$ increases linearly with $\rho$ even for relatively large values of $\rho(\approx 0.5 s)$ for $\rho>0$, whereas for $\rho<0$ the linearity holds only for small values of $\rho$.

\section{The orbital degenaracy at $\varepsilon_{0}=-2 s$}

A very interesting point of our phase diagram is at $\varepsilon_{0}=-2 s$, where the upper and lower bands touch. Then, depending on the system size $(L=8,12,16,20 \ldots$ with periodic boundary conditions or $L=6,10,14,18 \ldots$ with antiperiodic boundary conditions), the fully polarized ground state can be degenerate. This orbital degeneracy seems not to favor the highest spin states, as it was noticed e.g. in the case of Nagaoka ferromagnetism by Sütö. 25] To show this explicitly, we calculated the energy dispersion in the $S^{z}=S_{\max }-1$ subspace (see Fig. 10) and find that the difference between the energy $E$ of the groundstate in the $S_{\max }-1$ sector and the energy $E_{\mathrm{FM}}$ of the ferromagnetic state satisfies the following finite size scaling:

$$
E-E_{\mathrm{FM}}=\frac{C}{L^{2}}+\mathcal{O}\left(1 / L^{4}\right),
$$

with $C<0$, thus for any finite system the ground state has a total spin less than $S_{\max }$. 
It seems that this strange behavior is constrained to $\varepsilon_{0}=-2 s$ and away from it the $S_{\max }$ state is the ground state in the thermodynamic limit for $U>U_{c}$. To clarify this issue more precisely further work is needed.

\section{FERROMAGNETISM IN THE METALLIC CASE $(n<1 / 2)$}

Here we are mainly concerned with the case of large $U$ and $\varepsilon_{0}$. A nice feature of the effective Hamiltonian is that as far as the one-dimensional case is concerned, one can also discuss the metallic case corresponding to $n<1 / 2$. (A different way to show the stability of ferromagnetic state is given in Ref. [26].) Actually the metallic case is more interesting from a physical point of view than the insulating state. For $n<1 / 2$ all the terms in Eqs. (6)-(9) contribute. Although it looks complicated, the effective Hamiltonian in Eqs. (6)-(9) is similar to the $t-J$ model. Noting that $t^{\prime} / \varepsilon_{0}$ is a small expansion parameter, we can regard Eqs. (8) and (9) as a perturbation to Eqs. (6) and (7); the latter parts are essentially a spinless fermion Hamiltonian with nearest-neighbor hopping. The degeneracy with respect to spin can be lifted only after Eqs. (8) and (9) are introduced.

As far as $t^{\prime} / \varepsilon_{0}$ is small, we can apply the perturbation treatment which was used for the large $-U$ limit of the Hubbard model. 20 Just like in the latter case the ground state wave function $\left|\Psi_{g}\right\rangle$ is given as a direct product of spinless fermion wave function $\left|\Phi_{\mathrm{SF}}\right\rangle$ and spin wave function $|\chi\rangle$ :

$$
\left|\Psi_{g}\right\rangle=\left|\Phi_{\mathrm{SF}}\right\rangle \otimes|\chi\rangle
$$

To discuss the ground state of the effective Hamiltonian we take for $\left|\Phi_{\mathrm{SF}}\right\rangle$ the spinless fermion ground state of

$$
\mathcal{H}_{\mathrm{SF}}=s \sum_{j}\left(a_{j}^{\dagger} a_{j+1}+\text { H.c. }\right),
$$

where $a_{j}$ and $a_{j}^{\dagger}$ are spinless fermion annihilation and creation operators respectively. $|\chi\rangle$ has to be determined to minimize the spin-dependent part of the effective Hamiltonian. Taking the average of $\mathcal{H}_{\text {eff }}$ over $\left|\Phi_{\text {SF }}\right\rangle$ and picking up the spin-dependent part, we obtain

$$
\begin{aligned}
\left\langle\mathcal{H}_{\mathrm{eff}}\right\rangle_{\mathrm{SF}} & =J_{\mathrm{eff}} \sum_{i} \mathbf{S}_{i} \cdot \mathbf{S}_{i+1}, \\
J_{\mathrm{eff}} & =-4\left(\frac{t^{\prime}}{\varepsilon_{0}}\right)^{2}[s g(\nu)+t f(\nu)]+4 \frac{s^{2}}{U} h(\nu)
\end{aligned}
$$

where

$$
\begin{aligned}
& g(\nu)=\nu^{2}\left[1-\left(\frac{\sin (\pi \nu)}{\pi \nu}\right)^{2}\right] \\
& f(\nu)=\frac{1}{\pi^{2}} \sin (\pi \nu)[\pi \nu \cos (\pi \nu)-\sin (\pi \nu)], \\
& h(\nu)=\nu^{2}\left(1-\frac{\sin (2 \pi \nu)}{2 \pi \nu}\right) .
\end{aligned}
$$

The summation in Eq. 39 is taken over the squeezed spins. 20] $\nu$ is twice the density $n$ of electrons. For $0 \leq \nu \leq 1, g(\nu)$ and $h(\nu)$ are positive, while $f(\nu)$ is negative. Therefore, for positive $s$ and $t$, a competition occurs among three terms: the $s\left(t^{\prime} / \varepsilon_{0}\right)^{2}$ term favors ferromagnetism, whereas the $t\left(t^{\prime} / \varepsilon_{0}\right)^{2}$ and $s^{2} / U$ terms tend to destabilize it. Fig. 11 shows the sign of $J_{\text {eff }}$. For $0 \leq \nu \leq 1$, the ground state is ferromagnetic as long as $J_{\text {eff }}$ is negative.

Now let us turn to the excitation spectrum of the one spin flipped state in the same parameter region of $J_{\text {eff }} \ll t$. The low energy excitation of the spin part are the spin waves with momentum $q=2 \pi J / N_{e}$ and energy $\varepsilon(q)=J_{\text {eff }}(1-\cos q)$. The momentum of the spin part changes the boundary conditions of the spinless fermions, as noted by Woynarovich, 27 so that the momenta of spinless fermions are $(L / 2) k_{j}=2 \pi I_{j}+q$ with $I_{j}$ integers, and this phase shift appears in the energy and momentum of the spinless fermion part:

$$
\begin{aligned}
& E=J_{\mathrm{eff}}(1-\cos q)-2 s \sum_{j} \cos \frac{2 \pi I_{j}+q}{(L / 2)}, \\
& P=\sum_{j} k_{j}=2 \pi \frac{2}{L} \sum_{j} I_{j}+\frac{2 N_{e}}{L} q .
\end{aligned}
$$

In the thermodynamic limit, if the ground state of $q=0$ (i.e. of the highest spin state) is nondegenerate, then the energy $E(P)-E_{\mathrm{FM}}$ of the lowest lying excitations of the system is given by

$$
\omega(k)=J_{\mathrm{eff}}(1-\cos q)+\frac{2}{L} s q^{2} \frac{\sin \pi \nu}{\pi}
$$

where $k=\nu q+P_{\mathrm{FM}}$. Unlike the insulating case at $n=\frac{1}{2}$, this spin wave excitation is not separated any more by a finite gap from the Stoner continuum. We do not know the relevance of this fact to the stability of itinerant ferromagnetism in this case.

Depending on the number of particles, see Table. II], it can easily happen that the fully polarized state is degenerate. In this case of orbital degeneracy the energy of the spin wave with momentum $k=\nu q \pm\left|P_{\mathrm{FM}}\right|$ becomes

$$
\omega(k)=J_{\mathrm{eff}}(1-\cos q)+\frac{2}{L} s(q \mp \pi)^{2} \frac{\sin \pi \nu}{\pi}
$$

where we can clearly identify the competition between the kinetic energy gained by twisting the boundary conditions and the energy loss due to the spin wave. Actually, in the latter case, unless $J_{\text {eff }}>J_{c} \propto s \nu \sin \pi \nu$ , the minimum energy will occur for some finite $q$, and it prevents the highest spin state from being the ground state.

We have performed an exact diagonalization study of the model at $n=1 / 4$ (i.e. $\nu=1 / 2$ ) to determine the region where the fully polarized is stable against spin flip. The results are presented on Fig. 12. The behavior described above can be clearly seen for larger values of $U$ 
and $\left|\varepsilon_{0}\right|$, whereas near the flat band for $\varepsilon_{0}>\varepsilon_{\mathrm{FB}}$ the effect of orbital degeneracy is not so important . rahmanyam and X. Zotos. We also thank IDRIS (Orsay) for allocation of CPU time on the C94 and C98 CRAY supercomputers.

\section{ACKNOWLEDGMENTS}

We are especially thankful to $\mathrm{H}$. Tasaki for stimulating discussions. The authors enjoyed interesting discussions with P. Fazekas, K. Hallberg, J. Sólyom, V. Sub-

\section{APPENDIX: HIGHER ORDER TERMS IN THE SERIES EXPANSION}

Expansion of $K_{1}$ continues as:

$$
\begin{aligned}
K_{1}^{(4)}= & -\frac{16 s^{4}}{U^{3}}+\frac{4 t^{\prime 4}}{\varepsilon_{0}^{2} U}+\frac{8 t^{\prime 4}}{\varepsilon_{0}^{2}\left(2 \varepsilon_{0}+U\right)} \\
K_{1}^{(5)}= & -\frac{12 s^{3} t^{\prime 2}}{\varepsilon_{0}^{4}}+\frac{40 s t^{\prime 4}}{\varepsilon_{0}^{4}}+\frac{64 s^{3} t^{\prime 2}}{\varepsilon_{0} U^{3}}+\frac{32 s^{3} t^{\prime 2}}{\varepsilon_{0}^{2} U^{2}}+\frac{16 s t^{\prime 4}}{\varepsilon_{0}^{3} U} \\
K_{1}^{(6)}= & \frac{36 s^{2} t^{\prime 4}}{\varepsilon_{0}^{5}}+\frac{120 s^{6}}{U^{5}}-\frac{96 s^{2} t^{\prime 4}}{\varepsilon_{0}^{2} U^{3}}-\frac{64 s^{4} t^{\prime 2}}{\varepsilon_{0}^{2} U^{3}}-\frac{64 s^{4} t^{\prime 2}}{\varepsilon_{0}^{3} U^{2}}-\frac{96 s^{2} t^{\prime 4}}{\varepsilon_{0}^{3} U^{2}}-\frac{56 s^{4} t^{\prime 2}}{\varepsilon_{0}^{4} U}-\frac{16 s^{2} t^{\prime 4}}{\varepsilon_{0}^{4} U}-\frac{16 t^{\prime 6}}{\varepsilon_{0}^{4} U}+\frac{8 s^{4} t^{\prime 2}}{\varepsilon_{0}^{4}\left(\varepsilon_{0}+U\right)} \\
& +\frac{16 s^{2} t^{\prime 4}}{\varepsilon_{0}^{2}\left(2 \varepsilon_{0}+U\right)^{3}}+\frac{32 s^{2} t^{\prime 4}}{\varepsilon_{0}^{3}\left(2 \varepsilon_{0}+U\right)^{2}}+\frac{48 s^{\prime 4}}{\varepsilon_{0}^{4}\left(2 \varepsilon_{0}+U\right)}-\frac{96 \tilde{t}^{\prime}}{\varepsilon_{0}^{4}\left(2 \varepsilon_{0}+U\right)}, \\
K_{1}^{(7)}= & -\frac{84 \tilde{t}^{7}}{\varepsilon_{0}^{6}}-\frac{720 \tilde{t}^{7}}{\varepsilon_{0} U^{5}}-\frac{360 \tilde{t}^{7}}{\varepsilon_{0}^{2} U^{4}}+\frac{136 \tilde{t}^{7}}{\varepsilon_{0}^{3} U^{3}}+\frac{236 \tilde{t}^{7}}{\varepsilon_{0}^{4} U^{2}}+\frac{224 \tilde{t}^{7}}{\varepsilon_{0}^{5} U} \\
& -\frac{28 \tilde{t}^{7}}{\varepsilon_{0}^{5}\left(\varepsilon_{0}+U\right)}-\frac{32 \tilde{t}^{7}}{\varepsilon_{0}^{3}\left(2 \varepsilon_{0}+U\right)^{3}}-\frac{80 \tilde{t}^{7}}{\varepsilon_{0}^{4}\left(2 \varepsilon_{0}+U\right)^{2}}-\frac{32 \tilde{t}^{7}}{\varepsilon_{0}^{5}\left(2 \varepsilon_{0}+U\right)}
\end{aligned}
$$

where $\tilde{t}=t^{\prime}=s$. Contribution to $K_{2}$ starts in 4 th order:

$$
\begin{aligned}
& K_{2}^{(4)}=\frac{4 s^{4}}{U^{3}}, \\
& K_{2}^{(5)}=-\frac{16 s^{3} t^{\prime 2}}{\varepsilon_{0} U^{3}}-\frac{8 s^{3} t^{\prime 2}}{\varepsilon_{0}^{2} U^{2}} \\
& K_{2}^{(6)}=\frac{8 s^{2} t^{\prime 4}}{\varepsilon_{0}^{5}}-\frac{48 s^{6}}{U^{5}}+\frac{16 s^{4} t^{\prime 2}}{\varepsilon_{0}^{2} U^{3}}+\frac{24 s^{2} t^{\prime 4}}{\varepsilon_{0}^{2} U^{3}}+\frac{16 s^{4} t^{\prime 2}}{\varepsilon_{0}^{3} U^{2}}+\frac{24 s^{2} t^{\prime 4}}{\varepsilon_{0}^{3} U^{2}}+\frac{20 s^{4} t^{\prime 2}}{\varepsilon_{0}^{4} U}+\frac{4 s^{2} t^{\prime 4}}{\varepsilon_{0}^{4} U}+\frac{4 s^{4} t^{\prime 2}}{\varepsilon_{0}^{4}\left(\varepsilon_{0}+U\right)}+\frac{4 s^{2} t^{\prime 4}}{\varepsilon_{0}^{4}\left(2 \varepsilon_{0}+U\right)}, \\
& K_{2}^{(7)}=\frac{288 \tilde{t}^{7}}{\varepsilon_{0} U^{5}}+\frac{144 \tilde{t}^{7}}{\varepsilon_{0} U^{4}}-\frac{16 \tilde{t}^{7}}{\varepsilon_{0}^{3} U^{3}}-\frac{32 \tilde{t}^{7}}{\varepsilon_{0}^{4} U^{2}}-\frac{96 \tilde{t}^{7}}{\varepsilon_{0}^{5} U}-\frac{32 \tilde{t}^{7}}{\varepsilon_{0}^{5}\left(\varepsilon_{0}+U\right)}+\frac{16 \tilde{t}^{7}}{\varepsilon_{0}^{5}\left(2 \varepsilon_{0}+U\right)}, \\
& K_{3}^{(6)}=\frac{8 s^{6}}{U^{5}}, \\
& K_{3}^{(7)}=-\frac{48 \tilde{t}^{7}}{\varepsilon_{0} U^{5}}-\frac{24 \tilde{t}^{7}}{\varepsilon_{0}^{2} U^{4}}-\frac{8 \tilde{t}^{7}}{\varepsilon_{0}^{3} U^{3}}-\frac{12 \tilde{t}^{7}}{\varepsilon_{0}^{4} U^{2}} . \\
& \bar{K}_{2}^{(7)}=-\frac{8 \tilde{t}^{7}}{\bar{\varepsilon}_{0}^{6}}-\frac{8 \tilde{t}^{7}}{\bar{\varepsilon}_{0}^{5} U}+\frac{48 \tilde{t}^{7}}{\bar{\varepsilon}_{0}^{4}\left(2 \bar{\varepsilon}_{0}+U\right)^{2}}-\frac{48 \tilde{t}^{7}}{\bar{\varepsilon}_{0}^{5}\left(2 \bar{\varepsilon}_{0}+U\right)} .
\end{aligned}
$$

The coefficients $\bar{K}_{j}$ for $U,-\varepsilon_{0} \gg \tilde{t}$, where $\tilde{t}=s=t^{\prime}$ and we introduced the notation $\bar{\varepsilon}_{0}=-\varepsilon_{0}$, are:

$$
\begin{aligned}
& \bar{K}_{1}^{(6)}=\frac{20 \tilde{t}^{6}}{\bar{\varepsilon}_{0}^{5}}+\frac{24 \tilde{t}^{6}}{\bar{\varepsilon}_{0}^{4} U}+\frac{16 \tilde{t}^{6}}{\bar{\varepsilon}_{0}^{3}\left(2 \bar{\varepsilon}_{0}+U\right)^{2}}+\frac{16 \tilde{t}^{6}}{\bar{\varepsilon}_{0}^{4}\left(2 \bar{\varepsilon}_{0}+U\right)}, \\
& \bar{K}_{1}^{(7)}=\frac{72 \tilde{t}^{7}}{\bar{\varepsilon}_{0}^{6}}+\frac{32 \tilde{t}^{7}}{\bar{\varepsilon}_{0}^{5} U}-\frac{160 \tilde{t}^{7}}{\bar{\varepsilon}_{0}^{4}\left(2 \bar{\varepsilon}_{0}+U\right)^{2}}+\frac{352 \tilde{t}^{7}}{\bar{\varepsilon}_{0}^{5}\left(2 \bar{\varepsilon}_{0}+U\right)},
\end{aligned}
$$

* On leave from Research Institute for Solid State Physics, Budapest, Hungary.

E-mail: penc@mpipks-dresden.mpg.de

¥ E-mail: shiba@stat.phys.titech.ac.jp

$\S \quad$ E-mail: mila@irsamc2.ups-tlse.fr

[1] J. Kanamori, Prog. Theor. Phys. 30, 275 (1963).

[2] M. C. Gutzwiller, Phys. Rev. Lett. 10, 159 (1963).

[3] J. Hubbard, Proc. Roy. Soc. London A276, 238 (1993).

[4] Y. Nagaoka, Phys. Rev. 147, 392 (1966).

$$
\bar{K}_{2}^{(6)}=\frac{4 \tilde{t}^{6}}{\bar{\varepsilon}_{0}^{4} U}+\frac{16 \tilde{t}^{6}}{\bar{\varepsilon}_{0}^{4}\left(2 \bar{\varepsilon}_{0}+U\right)},
$$

[5] D. J. Thouless, Proc. Phys. Soc. London 86, 893 (1965). 
[6] T. Hanisch, B. Kleine, A. Ritzl and E. Müller-Hartmann, Annalen der Physik 4, 303 (1995); P. Wurth and E. Müller-Hartmann, Annalen der Physik 4, 144 (1995).

[7] W. von der Linden and D. M. Edwards, J. Phys. Cond. Matter 3, 4917 (1991).

[8] R. Strack and D. Vollhardt, Phys. Rev.Lett. 72, 3425 (1994).

[9] T. Yanagisawa and Y. Shimoi, Phys. Rev. Lett. 74, 4939 (1994), Phys. Rev. B48, 6104 (1993).

[10] H. Tasaki, Phys. Rev. Lett. 69, 1608 (1992); A. Mielke and H. Tasaki, Comm. Math. Phys. 158, 341 (1993).

[11] H. Tasaki, Phys. Rev. Lett. 73, 1158 (1994); H. Tasaki, to be published in J. Stat. Phys. .

[12] H. Tasaki, Phys. Rev. Lett. 75, 4678 (1995).

[13] K. Kusakabe and H. Aoki, Phys. Rev. Lett. 72, 144 (1994).

[14] A. Mielke, Phys. Lett. A174, 443 (1993) and references therein.

[15] E. Müller-Hartmann, J. Low Temp. Phys. 99, 349 (1995).
[16] M. W. Long, C. W. M. Castleton and C. A. Hayward, J. Phys. Cond. Matter 6, 481 (1994).

[17] E. H. Lieb, Phys. Rev. Lett. 62, 1201 (1989).

[18] M. Takahashi, J. Phys. Soc. Jpn. 51, 3475 (1982).

[19] B. S. Shastry, H. R. Krishnamurthy and P. W. Anderson, Phys. Rev. B41, 2375 (1990).

[20] M. Ogata and H. Shiba, Phys. Rev. B41, 2326 (1990); H. Shiba and M. Ogata: Int. J. Mod. Phys. B5, 31 (1991).

[21] K. Penc and F. Mila, Phys. Rev. B50, 11429 (1994).

[22] A. H. MacDonald, S. M. Girvin and D. Yoshioka, Phys. Rev. B 37, 9753 (1988).

[23] M. N. Barber and P. M. Duxbury, J. Stat. Phys. 29, 427 (1982).

[24] M. P. Gelfand, Solid State Comm. 98, 11 (1996).

[25] A. Sütő, Commun. Math. Phys. 140, 43 (1991), see also V. Subrahmanyam and F. Shi, unpublished.

[26] H. Tasaki, unpublished.

[27] F. Woynarovich, J. Phys. C: Solid State Phys 15, 85 (1982).

TABLE I. $\varepsilon_{\mathrm{FB}}$, constant $C$ and critical values of Coulomb repulsion $U$ for some selected values of $t^{\prime} / s$.

\begin{tabular}{cclcccccc}
\hline \hline & & \multicolumn{3}{c}{$\rho>0$} \\
$t^{\prime} / s$ & $\varepsilon_{\mathrm{FB}} / s$ & $C$ & $U_{D} / \rho$ & $U_{c} / \rho$ & $U_{c}^{\text {app }} / \rho$ & $U_{D} /|\rho|$ & $U_{c} /|\rho|$ & $U_{c}^{\text {app }} /|\rho|$ \\
\hline 4 & 14 & 0.00154 & 0.830 & 0.831 & 0.223 & 2.402 & 2.414 & 0.271 \\
2 & 2 & 0.0136 & 1.047 & 1.077 & 0.707 & 1.851 & 1.945 & 1 \\
$\sqrt{2}$ & 0 & 0.0302 & 1.235 & 1.355 & 1.155 & 1.527 & 1.715 & 1.406 \\
1 & -1 & 0.0557 & 1.476 & 1.860 & 1.789 & 1.277 & 1.555 & 1.505 \\
$1 / 2$ & -1.75 & 0.149 & 2.169 & 3.88 & 3.88 & 1.025 & 1.294 & 1.294 \\
\hline \hline
\end{tabular}

TABLE II. Momentum $P_{\mathrm{FM}}$ (with degeneracy) of the fully polarized state for periodic (PBC) and antiperiodic (APBC) boundary conditions, $t=0$.

\begin{tabular}{cccccc}
\hline \hline & \multicolumn{2}{c}{$\varepsilon_{0}>\varepsilon_{\mathrm{FB}}$} & \multicolumn{2}{c}{$\varepsilon_{0}<\varepsilon_{\mathrm{FB}}$} \\
$L / 2$ & $N_{e}$ & $\mathrm{PBC}$ & $\mathrm{APBC}$ & $\mathrm{PBC}$ & $\mathrm{APBC}$ \\
\hline even & even & $\pm \nu \pi$ & 0 & $\pm \nu \pi$ & 0 \\
even & odd & $\pi$ & $\pm(1-\nu) \pi$ & 0 & $\pm \nu \pi$ \\
odd & even & 0 & $\pm \nu \pi$ & $\pm \nu \pi$ & 0 \\
odd & odd & $\pm(1-\nu) \pi$ & $\pi$ & 0 & $\pm \nu \pi$ \\
\hline \hline
\end{tabular}




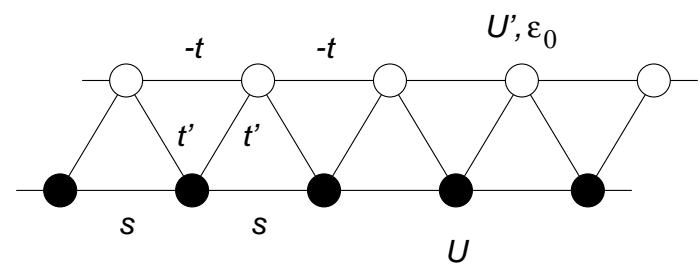

FIG. 1. Schematic representation on the 1D model. Solid circles stand for ' $c$ ' sites, and the open ones for ' $d$ ' sites.

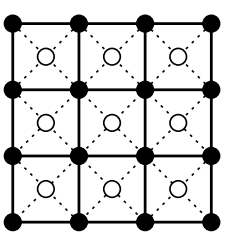

(a)

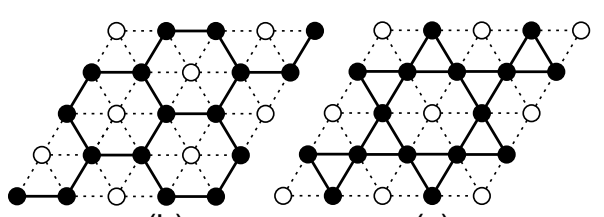

(b)

(c)
FIG. 2. Some possible realizations of the model in two dimensions: 'c' sites form square (a), honeycomb (b) and kagome (c) lattice.

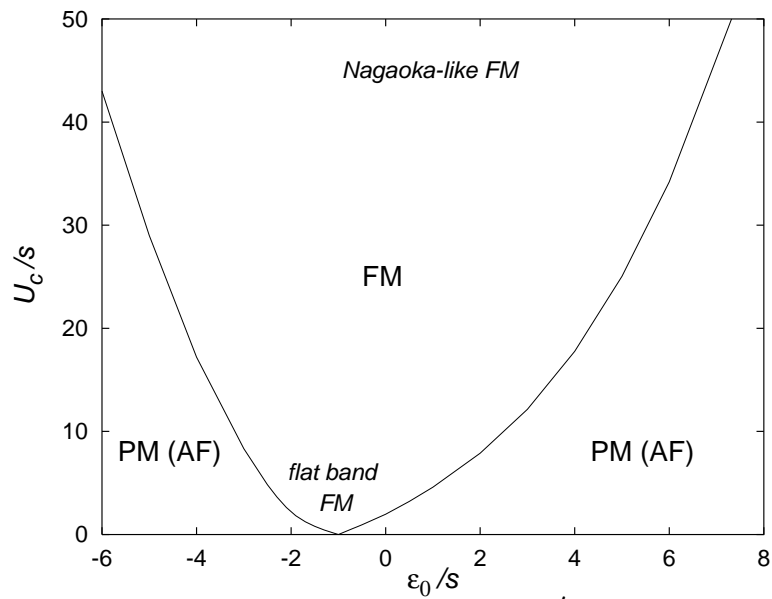

FIG. 3. Phase diagram for $t=0, s=t^{\prime}=1$ and quarter filling. A flat band is realized for $\varepsilon_{0}=-1$ and for $U=+\infty$ the model is ferromagnetic for any $\varepsilon_{0}$. For $U<U_{c}$ the system is paramagnetic, with strong antiferromagnetic correlations.

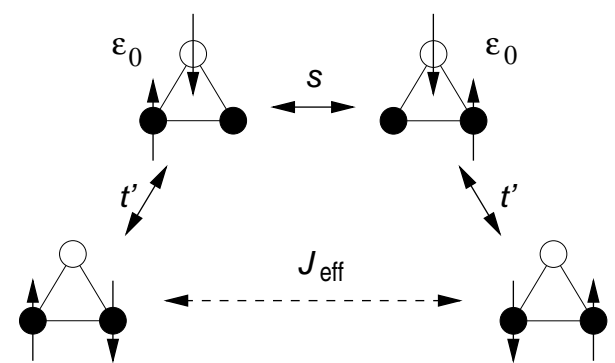

FIG. 4. The third order process leading to ferromagnetic effective exchange $J_{\text {eff }}=4 s t^{\prime 2} / \varepsilon_{0}^{2}$.

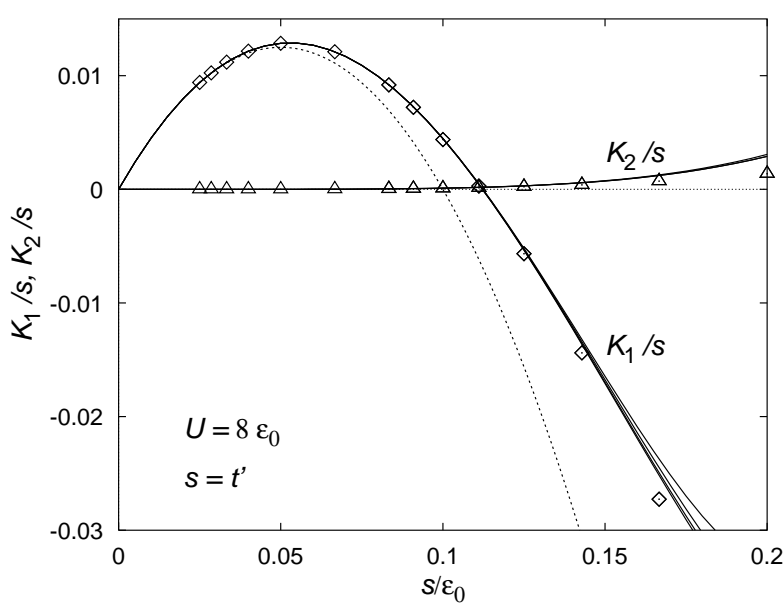

FIG. 5. The different Padé approximants for $K_{1}$ and $K_{2}$ are shown for $U / \varepsilon_{0}=8$. The series is convergent for $s / \varepsilon_{0} \sim 0.15$. The dashed line represents $K_{1}^{(2)}+K_{1}^{(3)}$. Note the excellent agreement with the values extracted from exact diagonalization data of a 16 site cluster (diamonds and triangles). The finite size effects are negligible.

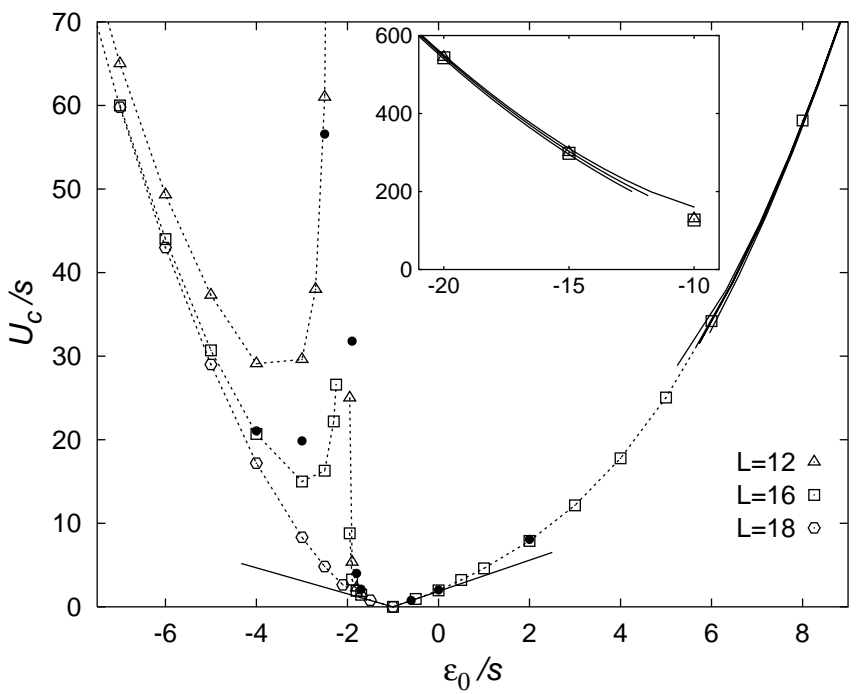

FIG. 6. Stability of the ferromagnetic state for $s=t^{\prime}$ and $t=0$, quarter filling. Solid lines for large values of $U_{c}$ come from Padé approximants of the perturbational expansion (main figure* and insert). Solid straight lines are the approximation near the flat band. Opens symbols are the data for local stability for system sizes 12,16 and 18 with periodic boundary condition. The finite size effect is negligible apart from the effect of degeneracy near $\varepsilon=-2 s$ for 12 and 16 site systems. Solid points are global stability data of the 16 site cluster. 

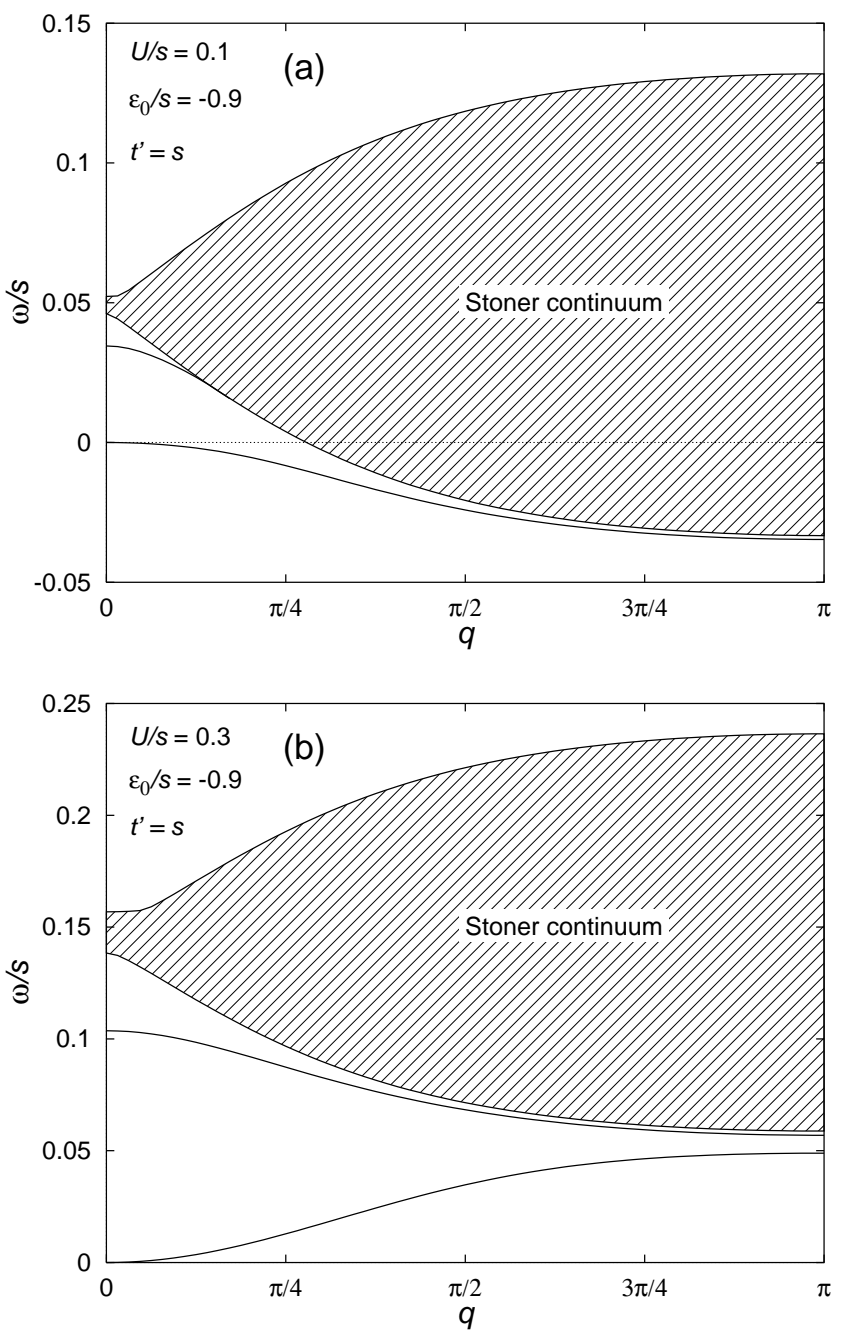

FIG. 7. The excitation spectra in the thermodynamic limit for some selected cases. (a) For small values of $U$ the fully polarized state is not stable, but already we can see a well defined magnon, which is very close to the particle-hole excitation continuum for $q=\pi$. (b) For larger values of $U$ we can see a normal ferromagnetic magnon dispersion relation, with the Stoner continuum pushed up to higher energies.

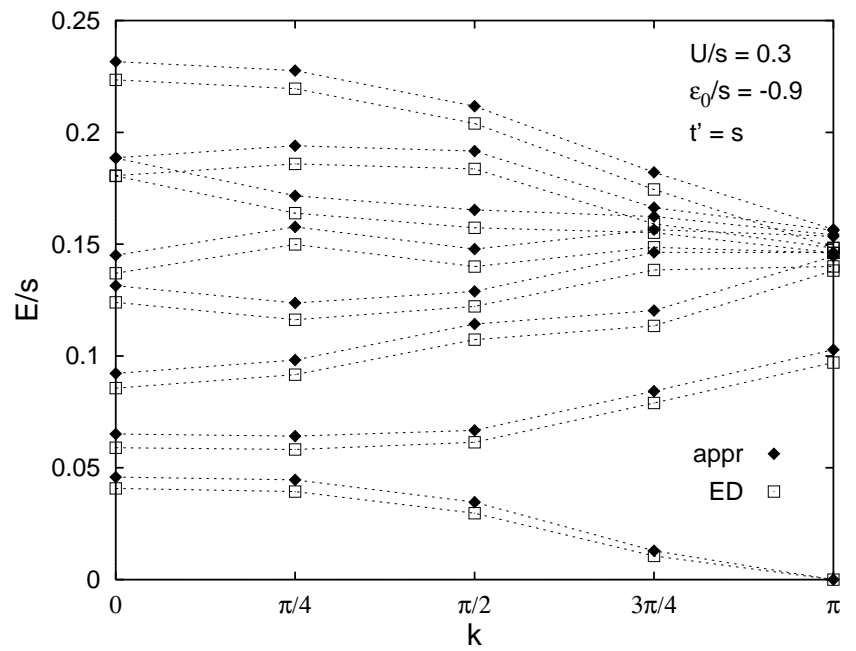

FIG. 8. The comparison of the excitation spectrum obtained from matrix $M_{p, p^{\prime}}(q)$ (solid diamonds) to exact diagolization of a 16 site cluster (open squares). Although $U / s=0.3$ is already comparable to the band gap, the errors are very small. The discrepancy is due interband scattering and it scales as $U^{2}$. Here $k=\pi-q$, where $\pi$ is the momentum of $|\mathrm{FP}\rangle$.
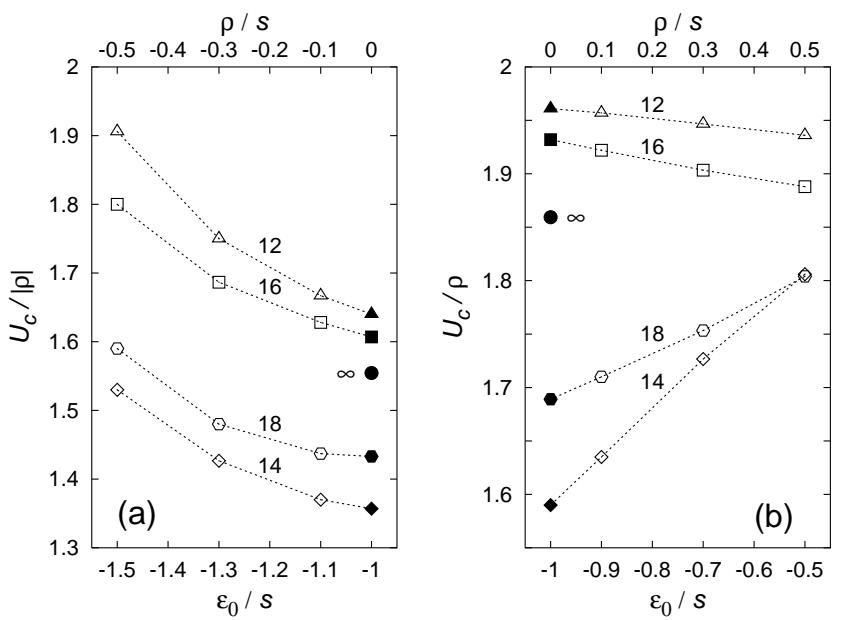

FIG. 9. $\quad U_{c} /|\rho|$ calculated from exact diagonalization (open symbols) and from diagonalization of matrix $M_{p, p^{\prime}}(\pi)$ (solid symbols) for system sizes $L=12,14,16$ and 18 with periodic boundary conditions, $t^{\prime}=s$ and $t=0$. The solid circle stands for the value of $U_{c}$ in the thermodynamic limit $L \rightarrow \infty$. 

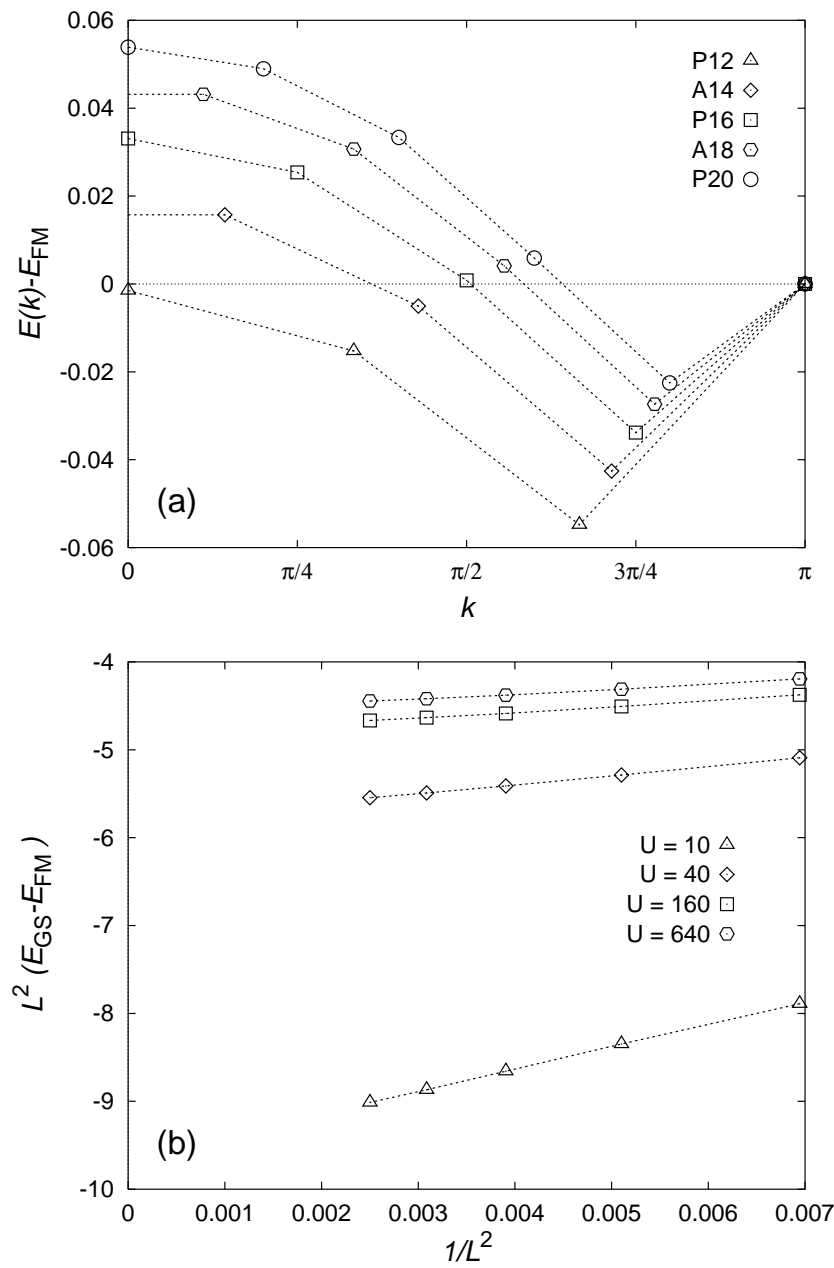

FIG. 10. (a) The dispersion of the lowest energy spin flipped state for $t^{\prime}=s=1, \varepsilon_{0}=-1$ and $t=0$ from exact diagonalization of 12,16 , and 20 site clusters with periodic $(\mathrm{P})$ and $L=14$ and 18 with antiperiodic (A) boundary conditions. Although the $S_{\max }$ state at $k=\pi$ is higher in energy, the dispersion has clearly ferromagnetic $k$ dependence. (b) The finite size scaling of the energy difference, $E_{\mathrm{GS}}$ is the lowest energy in the $S^{z}=S_{\max }-1$ subspace.

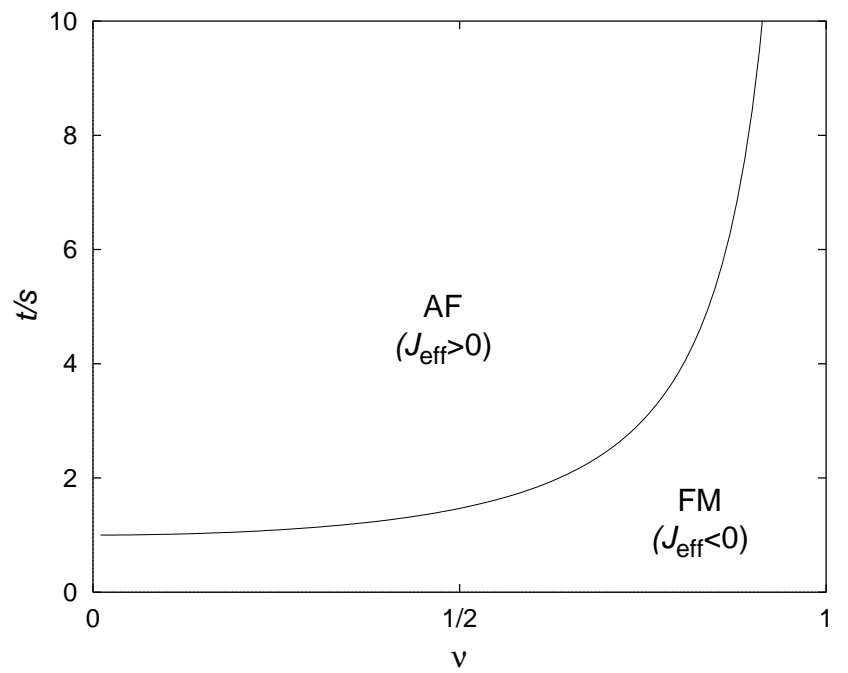

FIG. 11. For $U=+\infty$ and $t^{\prime} / \varepsilon_{0} \ll 1$ the sign of $J_{\text {eff }}$ and the nature of the ground state is determined by the ratio $t / s$. For negative $t$ it will always give a ferromagnetic effective coupling.

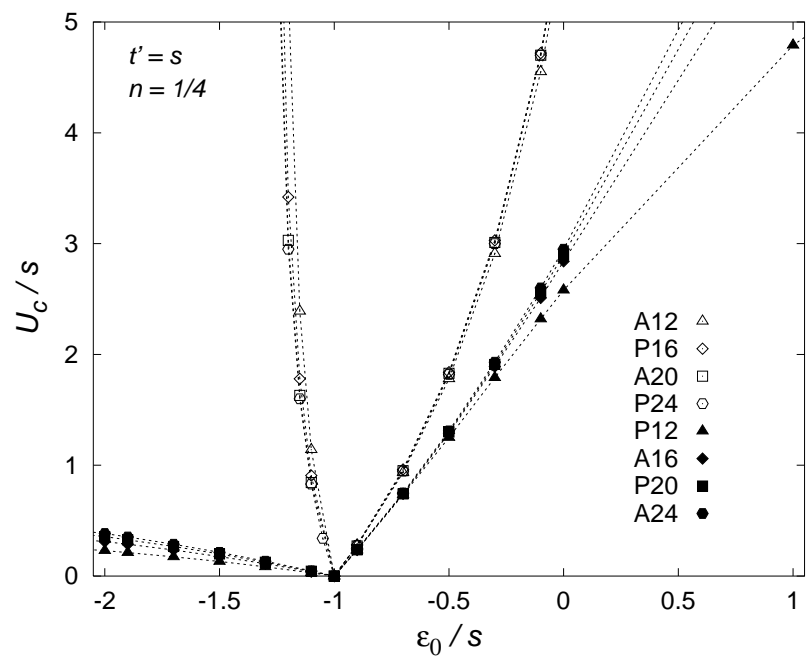

FIG. 12. Local stability for $n=1 / 4$ from exact diagonalization of $12,16,20$ and 24 site clusters with periodic $(\mathrm{P})$ or antiperiodic (A) boundary conditions $\left(t^{\prime}=s, t=0\right)$. The fully polarized state is degenerate for open symbols. Ferromagnetism is stable against single spin flip for $U>U_{c}$. 